\title{
TECHNOLOGICAL REQUIREMENTS OF NUCLEAR ELECTRIC PROPULSION SYSTEMS FOR FAST EARTH-MARS TRANSFERS
}

\author{
N. Bérend ${ }^{1}$, R. Epenoy ${ }^{2}$, E. Cliquet $^{3}$, J. Laurent-Varin ${ }^{3}$, \\ and S. Avril ${ }^{4}$
${ }^{1}$ ONERA - The French Aerospace Lab
Palaiseau 91761, France
${ }^{2}$ CNES - DCT
Toulouse 31401, France
${ }^{3}$ CNES - Launchers Directorate
Paris 75012, France
${ }^{4}$ ATOS ORIGIN
Toulouse 31024, France

\begin{abstract}
Recent advances in electric propulsion technologies such as magnetoplasma rockets gave a new momentum to the study of nuclear electric propulsion concepts for Mars missions. Some recent works have been focused on very short Earth-to-Mars transfers of about 40 days with high-power, variable specific impulse propulsion systems [1]. While the interest of nuclear electric propulsion appears clearly with regard to the payload mass ratio (due to a high level of specific impulse), its interest with regard to the transfer time is more complex to define, as it depends on many design parameters. In this paper, a general analysis of the capability of nuclear electric propulsion systems considering both criteria (the payload mass ratio and the transfer time) is performed, and the technological requirements for fast Earth-Mars transfers are studied. This analysis has been performed in two steps. First, complete trajectory optimizations have been performed by CNES-DCT in order to obtain the propulsion requirements of the mission for different technological hypotheses regarding the engine technology (specific impulse levels and the throttling capability) and different mission requirements. The methodology used for designing fuel-optimal heliocentric trajectories, based on the Pontryagin's Maximum Principle will be presented. Trajectories have been computed for various power levels combined with either variable or fixed $I_{\mathrm{sp}}$. The second step consisted in evaluating a simpler method that could easily link the main mission requirements
\end{abstract}


(the transfer time and the payload fraction) to the main technological requirements (the specific mass of the power generation system and the structure mass ratio of the whole vehicle, excluding the power generation system). Indeed, for power-limited systems, propulsion requirements can be characterized through the "trajectory characteristic" parameter, defined as the integral over time of the squared thrust acceleration. Technological requirements for the vehicle can then be derived from the propulsion requirements using a simplified performance model designed by Onera [2]. This model yields the optimum vehicle design in terms of the payload mass ratio as well as the theoretical upper limit of the power source's specific mass as a function of the transfer time. Both studies show that the key to very fast Earth-Mars transfers (40 days, or less) is the reduction of the power source specific mass below $1 \mathrm{~kg} / \mathrm{kW}$. On-going French studies [3] tend to show that specific masses of nuclear reactors for exploration mission are expected to be much higher, even at very high power levels, so this requirement is unlikely to be met at short or medium term. Finally, a synthesis of these results will outline the performance of a nuclear electric propulsion system for fast Earth-Mars transfer that could be achievable considering "reasonably optimistic" hypotheses for the specific mass of the power generator.

\title{
GLOSSARY
}

\author{
IMLEO Initial Mass in Low-Earth Orbit \\ LEO Low-Earth Orbit \\ MHD Magnetohydrodynamic \\ MPD Magnetoplasmadynamic \\ PMAD Power Management and Distribution \\ VASIMR VAriable Specific Impulse Magnetoplasma Rocket
}

\section{INTRODUCTION}

One of the most challenging aspects of human Mars missions is the long duration of the transfers. The Earth-to-Mars transfer time is typically about 6 months or greater in scenarios using the conventional chemical or nuclear thermal propulsion.

Previous studies tend to show that nuclear electric propulsion is a good candidate for manned Mars missions, allowing increased payload ratios, more flexible departure window opportunities and potentially, shorter durations compared to more conventional propulsion [4-7].

In this paper, the feasibility of faster interplanetary transfers using the nuclear electric propulsion and, generally speaking, power-limited propulsion systems is discussed. 
In section 2, the method and the results obtained by CNES-DCT concerning fuel-optimal trajectory computations are presented. This study made it possible to evaluate precisely the best payload ratio that can be achieved for a given transfer time (39, 60, 90, 120, and 180 days) with various power levels using either fixed or variable specific impulse.

In section 3, a generic analysis of Power-Limited systems performance using a very simple model is presented. This analysis emphasizes the importance of the specific mass of the power and propulsion system.

In section 4, the levels of achievable specific mass for the power and propulsion system depending of the technology are discussed. Finally, in section 5, different Earth-to-Mars transfer scenarios, including an improved version of the fast transfer mission studied by Ad Astra for the VASIMR (VAriable Specific Impulse Magnetoplasma Rocket) concept (cf. [1] and [8]) and other scenarios based on the hypotheses of achievable specific mass defined in section 4 are discussed.

\section{TRAJECTORY OPTIMIZATION FOR FAST EARTH-MARS TRANSFERS WITH HIGH POWER NUCLEAR ELECTRIC PROPULSION}

\subsection{Methodology}

An Earth-Mars trajectory is commonly split into three phases: two planetocentric phases (one for the Earth's departure, the other for Mars arrival) and one heliocentric phase. For the present work, only the first planetocentric phase and the heliocentric phase are considered.

Two cases were assessed: a case of a thruster with a fixed specific impulse equal to $5000 \mathrm{~s}$ and a case of a thrusters with a specific impulse that can be varied between $I_{\mathrm{sp}_{1}}=3,000 \mathrm{~s}$ and $I_{\mathrm{sp}_{2}}=30,000 \mathrm{~s}$. However, the constant maximum thrust $\left(I_{\mathrm{sp}}\right.$ fixed at its lower value $I_{\mathrm{sp}_{1}}$ ) is used for the first planetocentric phase, even in the case of a variable specific impulse thruster. It should be noted that these $I_{\mathrm{sp}}$ values, for both the fixed $I_{\mathrm{sp}}$ and the variable $I_{\mathrm{sp}}$ cases, correspond to optimistic hypotheses. The value of $5000 \mathrm{~s}$ of $I_{\mathrm{sp}}$ could be achieved by magnetoplasmadynamic (MPD) thrusters fed with hydrogen or even Lithium [9] and the variable $I_{\mathrm{sp}}$ hypotheses corresponds to Ad-Astra values for the VASIMR engine [1]. Those performances are not flight proven, and both thrusters have only been ground tested at power levels of a few hundreds of kilowatts (up to a few megawatts for MPDs). However, although these technologies are far from being "on the shelf," today these hypotheses are considered to be reasonably optimistic in a long term perspective and regarding the fact that such a mission also implies a high power nuclear reactor which also has a low technology readiness level today (this topic is further discussed in section 4). 
Optimal tuning of the specific impulse is not classical, thus, hereafter, the necessary optimality conditions induced by this additional degree of freedom during heliocentric phase are presented.

\subsubsection{Dynamic model}

The equations describing the dynamic model are as follows:

$$
\begin{aligned}
\dot{r}(t) & =v(t) \\
\dot{v}(t) & =-\mu \frac{r(t)}{\|r(t)\|^{3}}+\frac{T(t)}{m} U(t) ; \\
\dot{m}(t) & =-\frac{T(t)}{g_{0} I_{\mathrm{sp}}(t)}
\end{aligned}
$$

where $r(t)$ is the spaceship position vector at time $t ; v(t)$ is the spaceship velocity vector at time $t ; m(t)$ is the spaceship mass at time $t ; T(t)$ is the thrust value at time $t ; U(t)$ is the thrust direction vector at time $t(\|U(t)\|=1) ; I_{\mathrm{sp}}$ is the specific impulse at time $t$; and $\mu$ and $g_{0}$ are the gravitational constants of the Sun and, respectively, the acceleration due to the gravity at the sea level $g_{0}=9.80665 \mathrm{~m} / \mathrm{s}^{2}$.

The control variables are then $T(t), U(t)$, and $I_{\mathrm{sp}}(t)$. These variables have to be optimized in the feasible control space $F$ defined as follows:

$$
F=\left\{\left(T, U, I_{\mathrm{sp}} / T \in\left[0, \frac{2 \eta P_{e}}{g_{0} I_{\mathrm{sp}}}\right], U \in \mathfrak{R}^{3},\|U\|=1, I_{\mathrm{sp}} \in\left[I_{\mathrm{sp}_{1}}, I_{\mathrm{sp}_{2}}\right]\right\}\right.
$$

where $\eta \in] 0,1]$ is the efficiency coefficient of the engine; and $P_{e} \in \mathfrak{R}^{+}$is the electrical power.

\subsubsection{Pontryagin's maximum principle}

According to the maximum principle, the optimal control is given by the minimizing control of the Hamiltonian:

$$
H=\xi_{r}^{T} v-\mu \frac{\xi_{v}^{T} r}{\|r\|^{3}}+T\left[\frac{\xi_{v}^{T} U}{m}-\frac{\xi_{m}}{g_{0} I_{\mathrm{sp}}}\right]
$$

where $\xi_{r}, \xi_{v}$, and $\xi_{m}$ are the co-states associated with the position $r$, the velocity $v$, and the mass $m$, respectively.

The well-known adjoint equations of the co-states are not presented.

Remark. In the minimization process, undefined situations corresponding to singular arcs are avoided by assigning arbitrary values to undefined control variables. 
Table 1 Optimal values of control variables

\begin{tabular}{|c|c|c|c|c|}
\hline$\xi_{v}$ & $\xi_{m}$ & $\bar{U}$ & $I_{\mathrm{sp}}$ & $T$ \\
\hline \multirow{3}{*}{$=0$} & $>0$ & \multirow{3}{*}{$(1,0,0)$} & $I_{\mathrm{sp}_{1}}$ & $T_{\max }$ \\
\hline & $=0$ & & $I_{\mathrm{sp}_{1}}$ & $T_{\max }$ \\
\hline & $<0$ & & $I_{\mathrm{sp}_{2}}$ & 0 \\
\hline \multirow[b]{3}{*}{$\neq 0$} & $>0$ & \multirow[b]{3}{*}{$\frac{\xi_{v}}{\mid \xi_{v} \|}$} & $I_{\mathrm{sp}_{1}}$ & $T_{\max }$ \\
\hline & $=0$ & & $I_{\mathrm{sp}_{1}}$ & $T_{\max }$ \\
\hline & $<0$ & & $\begin{array}{l}\min \left(I_{\mathrm{sp}_{2}},\right. \\
\left.\max \left(I_{\mathrm{sp}_{1}},-\frac{2 m \xi_{m}}{g_{0}\left\|\xi_{v}\right\|}\right)\right)\end{array}$ & $\left\{\begin{array}{l}\frac{2 \eta P_{e}}{g_{0} I_{\mathrm{sp}}} \quad \text { if } \\
-\frac{\left\|\xi_{v}\right\|}{m}-\frac{\xi_{m}}{g_{0} I_{\mathrm{sp}}}<0 \\
0 \quad \text { else }\end{array}\right.$ \\
\hline
\end{tabular}

Remark: $T_{\max }=2 \eta P_{e} /\left(g_{0} I_{\mathrm{sp}_{1}}\right)$.

Table 1 gathers the optimal values of control variables $\left(T, U\right.$, and $\left.I_{\mathrm{sp}}\right)$ as the functions of co-states values.

\subsection{Results}

After the first trajectory computation dedicated to the cross-check of AARC (AdAstra Rocket Company) results that are briefly presented in paragraph 2.2.1, some other calculations associated with different hypotheses concerning IMLEO (Initial Mass In Low-Earth Orbit) and different values of the transfer duration, plus additional cases with constant $I_{\mathrm{sp}}$ were carried out in order to evaluate the interest of variable $I_{\mathrm{sp}}$ thrusters technologies vs. fixed ones (like, for example, MPD thrusters).

\subsubsection{Cross-check of Ad-Astra scenario}

Before going into computations of various trajectories for fast manned Mars missions using high power electric propulsion, the cross-checking AARC results for the "39 days-200 MW scenario" given in [8] was made.

The main hypothesis concerns a departure from the circular Low-Earth Orbit (LEO) of 1000-kilometer altitude, the relative velocity with respect to the Earth (V-Infinity) equal to $2.5 \mathrm{~km} / \mathrm{s}$ at the Earth's departure, and the heliocentric phase with variable specific impulse (the latter being considered as a control variable).

The departure date from LEO is assumed to be July 7, 2018 and the initial ship mass is equal to $600 \mathrm{t}$.

Concerning the geocentric phase, the computations lead to a very similar path as AARC one's. Indeed, the computed time to escape, i. e., to reach a 


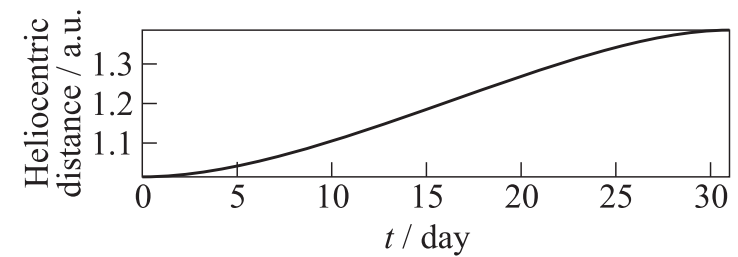

(a)

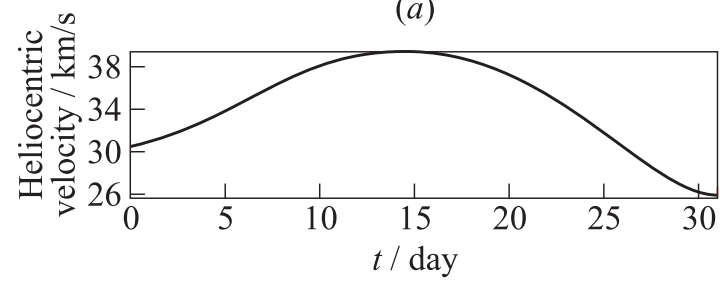

(b)

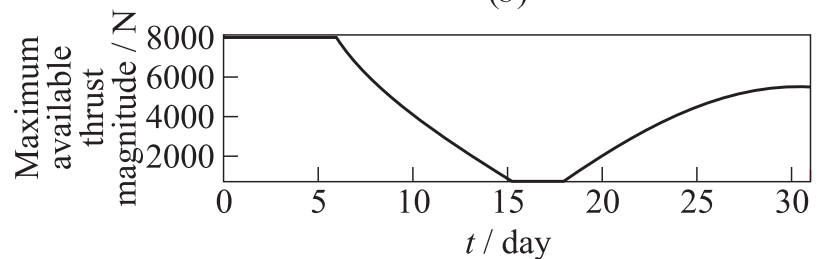

(c)

Figure 1 Heliocentric distance (a), heliocentric velocity $(b)$, and value of $T(c)$ over time

$5.5 \mathrm{~km} / \mathrm{s} \mathrm{V}$-Infinity, is equal to 7.8 days (compared to 8 days in [8]), and the propellant mass consumed is equal to $162.9 \mathrm{t}$ (compared to $165 \mathrm{t}$ in [8]).

For the heliocentric arc, in order to have exactly the same initial conditions as in [5], an initial mass is assumed to be equal to $435 \mathrm{t}$. As a consequence, this value is not consistent with the propellant consumption of the geocentric phase. According to [1], the Earth's departure date is July 15, 2018, the initial V-Infinity is equal to $5.5 \mathrm{~km} / \mathrm{s}$, the heliocentric transfer duration is equal to 31 days, and the final V-Infinity at Mars arrival is equal to $6.8 \mathrm{~km} / \mathrm{s}$. After computation, the optimal trajectory with a final mass equal to $146.5 \mathrm{t}$ was obtained while Ad Astra obtained $140 \mathrm{t}$ in [8].

As can be observed in Fig. 1, the optimal thrust level $T$ varies over time and is very similar to the optimal AARC law (see [8]). It can also be noted that the engine is always operating (no coast arcs).

The specific impulse varies during the flight according to Fig. 2.

This concludes the cross-check by recovering the results found by AARC in [8]. Thus, it is confirmed that to be able to perform a 39-day trajectory, the requirements on the propulsion system in terms of power and specific mass 


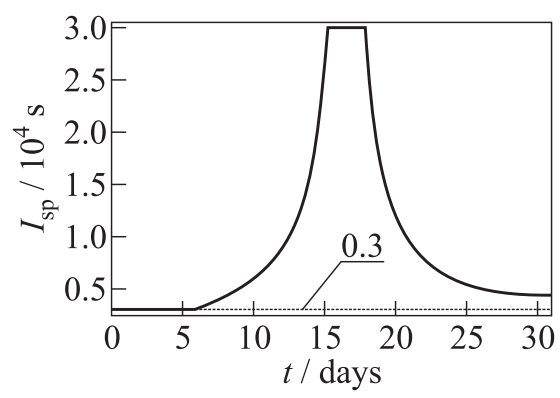

Figure 2 Time variations of the specific impulse

(respectively, $200 \mathrm{MW}$ and lower than $1 \mathrm{~kg} / \mathrm{kW}$ ) are very challenging. This fact is fully recognized by AARC [10].

\subsubsection{Results of the parametric study}

In order to optimize the most of parameters and to identify what are the best compromises, a decision was made to compute optimal trajectories based on the following large set of hypothesis:

- IMLEO: from 100 to $500 \mathrm{t}$;

- initial V-Infinity at Earth's departure: from 0 to $6 \mathrm{~km} / \mathrm{s}$;

- level of nuclear reactor power: 5, 10, 20, and $50 \mathrm{MW}$;

- total transfer duration: 60, 90, and 120 days (plus one computation with 180 days).

Note that V-infinity at Mars arrival is equal to $6.8 \mathrm{~km} / \mathrm{s}$ in all cases.

Moreover, in order to estimate the gain obtained by using variable specific impulse propulsion, some trajectory optimizations with a constant $I_{\mathrm{sp}}$ equal to $5000 \mathrm{~s}$ were performed (such fixed specific impulse could possibly be achievable with MPD technology and hydrogen). Those results could be completed with other levels of fixed $I_{\mathrm{sp}}$, and it is foreseen to perform such calculation in a further study.

Figure 3 presents the final mass obtained at Mars arrival for the variable specific impulse propulsion and for a set of different cases defined above. Let outline here that certain cases (combinations of IMLEO, initial V-Infinity, transfer duration, etc.) do not lead to a feasible trajectory (for example, the final mass may be negative), and consequently, they are not presented in Fig. 3. 


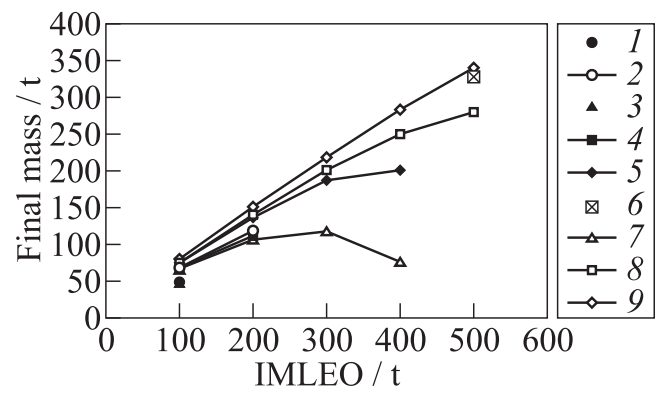

Figure 3 Final mass at Mars for VASIMR ${ }^{\circledR}$ propulsion: $1-P=5 \mathrm{MW}, 120$ days; $2-P=10 \mathrm{MW}, 120$ days; $P=20 \mathrm{MW}: 3-60$ days, $4-90,5-120$, and $6-$ 180 days; and $P=50 \mathrm{MW}: 7-60$ days, $8-90$, and $9-120$ days

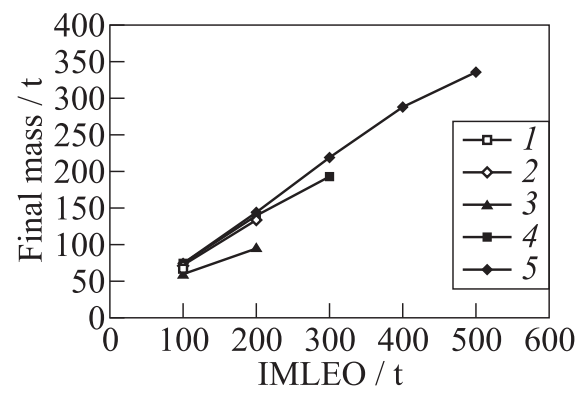

Figure 4 Final mass at Mars for fixed 5000-second specific impulse: $P=20 \mathrm{MW}$ : $1-90$ days and $2-120$ days; and $P=50$ MW: $3-60$ days, $4-90$, and $5-$ 120 days

Remark. The best value for the initial V-Infinity among those computed (leading to the maximum final mass at Mars) is chosen for all the cases described in Fig. 5 below. This best value is case dependent. Indeed, when the initial V-Infinity increases (from 0.0 to $6.0 \mathrm{~km} / \mathrm{s}$ ), the fuel consumption associated with the heliocentric phase decreases but, on the other hand, the fuel consumption associated with the escape phase increases. As a consequence, the best V-Infinity is in general an intermediate value that depends on the case under consideration.

In case of the 5000 -second $I_{\mathrm{sp}}$ MPD propulsion, the corresponding final masses are given in Fig. 4.

The final masses at Mars obtained (see Figs. 3 and 4) include the vehicle structure mass, the tanks mass, the thrusters mass, and the power generation system mass. Section 4 will allow to identify the achievable values for those masses and thus to evaluate the real payload associated with these figures. 


\section{GENERIC RESULTS FOR POWER-LIMITED PROPULSION SYSTEMS}

\subsection{Problem Definition and Hypotheses}

In this section, the relation between the transfer time, the performance (in terms of payload mass ratio), and technological hypotheses for a power-limited propulsion system is analyzed.

In such systems, the energy used for rocket propulsion (i. e., used to accelerate and eject the mass of the jet) comes from of a power source delivering a power level $P_{e}$. We denote $\eta$ the global efficiency of the system, which links $P_{e}$ to the power actually used for the propulsion, as shown in

$$
\frac{1}{2} \dot{m} V_{E}^{2}=\eta P_{e}
$$

where $\dot{m}$ is the mass flow rate of the ejected mass and $V_{E}$ is the ejection velocity.

Depending on the thruster technology, the mass flow-rate and the ejection velocity (i. e., the thrust and specific impulse levels) can be adjusted during the transfer or remain fixed.

According to the classic result [11], the rocket equation for a Power-Limited system with a fixed global efficiency can be expressed as

$$
\int_{0}^{t_{f}} \gamma_{T}^{2} d t=2 \eta P_{e}\left(\frac{1}{M_{f}}-\frac{1}{M_{i}}\right)
$$

where $\gamma_{T}$ is the thrust acceleration, which is a function of the time $(t)$, and $M_{i}$ and $M_{f}$ are the total initial and final masses, respectively. This relation is valid for any thrust and specific impulse law, provided it satisfies Eq. (1). However, it depends on the simplifying hypothesis that the global efficiency $\eta$ remains constant throughout the transfer regardless of the current level of specific impulse.

In the same way as the classic rocket equation (for "Constant Ejection Velocity" propulsion systems), Eq. (2) can be interpreted as a relation between the mission propulsion requirements for the considered trajectory profile (represented by the left-hand term) and the system design (represented by the righthand term). The left-hand term of Eq. (2) is comparable to the "ideal velocity" (also called "propulsive velocity increment") of the classic rocket equation, as it depends only on the acceleration profile. For the purpose of convenience, this term $\lambda$ will be denoted and referred to as the "trajectory characteristic parameter:"

$$
\lambda=\int_{0}^{t_{f}} \gamma_{T}^{2} d t .
$$


The unit of $\lambda$ is $\mathrm{m}^{2} / \mathrm{s}^{3}$, which is equivalent to $\mathrm{W} / \mathrm{kg}$.

Equation (2) shows that, in the trajectory optimization problem for a vehicle with a given total initial mass $\left(M_{i}\right)$, the objective of maximizing the final mass (i. e., maximizing the payload mass) amounts to minimizing $\lambda$. Thus, in the same way as the propulsive velocity increment for classic rocket systems (which should be minimized for the best performance), the minimum value of $\lambda$ characterizes up to a certain extent - the mission requirements for a given scenario. Also, Eq. (2) can be used in the same way as the classic rocket equation to perform a preliminary vehicle design considering a pre-defined estimation of the mission requirements (represented by $\lambda$ ). This analysis can be based on pre-calculated tables of $\lambda$ (cf., for instance, [11] and [12]).

The validity of this approach depends on the assumption that the minimum value of $\lambda$ for a given mission is invariant (or nearly invariant) to the vehicle characteristics, so that the value of $\lambda$ can be reused from one vehicle design to another, considering the same mission and the same range of $I_{\mathrm{sp}}$ variation. Actually, $\lambda$ cannot be completely invariant to the vehicle's total mass and power level, because these parameters have some influence on the acceleration level $\gamma_{T}$, but this dependence is greatly reduced for variable thrust systems, especially when the specific impulse can vary in a large domain (which is the case in this paper). So, for this kind of systems, this assumption appears to be acceptable, and a precalculated $\lambda$ obtained through a single trajectory optimization for a given vehicle design can be considered to have the correct order of magnitude for a more general mission analysis. This approach has been observed to be acceptable for the fixed thrust propulsion systems also (the minimum value of $\lambda$ has been shown to be nearly invariant to the size of the power source, cf. $[13])$.

When considering a given mission scenario (e.g., an Earth-to-Mars transfer with prescribed initial and final conditions), the value of $\lambda$ depends on the transfer time only and a law for $\lambda$ as a function of the transfer time (cf. examples in [11] and [12]) can be defined. Thus, Eq. (2) links the transfer time (through $\lambda$ ) to the vehicle's masses and power.

In this section, Eq. (2) is used to study the optimum vehicle design with regard to the objective of maximizing the payload mass ratio for a given transfer time, and technological requirements for fast Earth-to-Mars transfers are derived. For this study, the vehicle's mass budget is defined through the simplified model described in Table 2 .

Remark. Other authors have studied similar problems but with a different model for the mass budget and/or a different objective function (cf. [11], [12], and [14]).

This mass budget model uses the following parameters, which characterize the vehicle's technology: 
Table 2 Mass budget elements and model

\begin{tabular}{lc}
\hline Mass budget element & Model \\
\hline Propellant $\left(M_{P}\right)$ & $M_{P}=M_{i}-M_{f}$ \\
Tank structure $\left(M_{S}\right)$ & $M_{S}=k M_{P}$ \\
Power and propulsion system $\left(M_{T}\right)$ & $M_{T}=\alpha P_{e}$ \\
Payload $\left(M_{\mathrm{pl}}\right)$ & $M_{\mathrm{pl}}=M_{f}-M_{S}-M_{T}$ \\
\hline
\end{tabular}

- the tank structural mass ratio $(k)$, defined by

$$
k=\frac{M_{S}}{M_{P}}
$$

- the specific mass of the power and propulsion system $(\alpha)$, defined by

$$
\alpha=\frac{M_{T}}{P_{e}} .
$$

\subsection{Optimum Vehicle Design for a Given Transfer Time}

By transforming Eq. (2), it can be shown that the payload mass ratio $\left(M_{\mathrm{pl}} / M_{i}\right)$ can be expressed as a function of the power-to-initial mass ratio $P_{e} / M_{i}$ (cf. [2]):

$$
\frac{M_{\mathrm{pl}}}{M_{i}}=\left(\frac{2 \eta P_{e} / M_{i}-k \lambda}{2 \eta P_{e} / M_{i}+\lambda}\right)-\alpha \frac{P_{e}}{M_{i}} .
$$

Using Eq. (3), it can be shown (cf. [2]) that the payload mass ratio is maximized for:

$$
\frac{P_{e}}{M_{i}}=\sqrt{\frac{\lambda(1+k)}{2 \eta \alpha}}-\frac{\lambda}{2 \eta} .
$$

The maximum payload mass ratio obtained with this value is:

$$
\frac{M_{\mathrm{pl}}}{M_{i}}=\frac{\alpha \lambda}{2 \eta}-\sqrt{\frac{2 \alpha \lambda(1+k)}{\eta}}+1 .
$$

Solving Eq. (5) for $\alpha$, one obtains the following expression of the specific mass as a function of the maximum payload mass ratio $\left(M_{\mathrm{pl}} / M_{i}\right)$ and the trajectory characteristic parameter $\lambda$ :

$$
\alpha=\frac{2 \eta}{\lambda}\left(\sqrt{1+k}-\sqrt{k+\frac{M_{\mathrm{pl}}}{M_{i}}}\right)^{2} .
$$


Equation (6) actually yields the maximum specific mass a vehicle can have for a given mass ratio and a given trajectory characteristic parameter $\lambda$ (hence, a given transfer time $t_{f}$, according to the hypotheses exposed previously). Indeed, it can be shown that the value of $\alpha$ obtained through the solving of Eq. (5) (which characterizes the vehicle design with the maximum payload mass-ratio for a given $\alpha$ ) is the same value that can be obtained by maximizing $\alpha$ in the general case (i. e., as defined by Eq. (3) for a given payload mass ratio) with respect to $\left(P_{e} / M_{i}\right)$.

An additional result derived from Eq. (6) is the theoretical maximum value of $\alpha$ for the "limit" case of a null payload (i. e., null payload mass ratio):

$$
\alpha_{\text {limit }}=\frac{2 \eta}{\lambda}(\sqrt{1+k}-\sqrt{k})^{2} .
$$

\subsection{Application to Earth-to-Mars Transfer}

In this section, Eq. (6) is used to define the specific mass requirement as a function of the prescribed payload mass ratio and transfer time, for an Earth (LEO) to Mars transfer with a VASIMR-like propulsion system (cf. [1, 8, 10, 15, $16]$ ). For this study, the following hypotheses (which are the same as in [8]) are considered:

- tank structural mass ratio: $k=0.1$;

- global efficiency: $\eta=0.6 ;$ and

- $I_{\mathrm{sp}}$ variation range: $3,000-30,000 \mathrm{~s}$.

It should be noted that these values appear to be optimistic, especially considering the fact that $k$ and $\eta$ are considered as constants in the problem (cf. the list of assumptions detailed below). However, it can be observed from Eqs. (6) and (7) that the uncertainty on these data will not change the order of magnitude of the results.

In order to introduce the transfer time in the calculation, the following simple model for the estimation of $\lambda$ as a function of the transfer time is presented. This model has been obtained through least-square fitting of a set of VASIMR mission analysis results from [15] (after the calculation of the $\lambda$ data using Eq. (2)):

$$
\lambda\left(t_{f}\right) \approx 5.2220 \cdot 10^{7} t_{f}^{-2.9183} .
$$

The units in this model are: $t_{f}$ in days and $\lambda$ in $\mathrm{m}^{2} / \mathrm{s}^{3}$.

By combining Eqs. (8) and (6), the specific mass requirement for transfer times between 40 and 180 days, and for different payload mass ratios between 0 (which represents the theoretical feasibility "limit" for the mission) and 0.6 were 


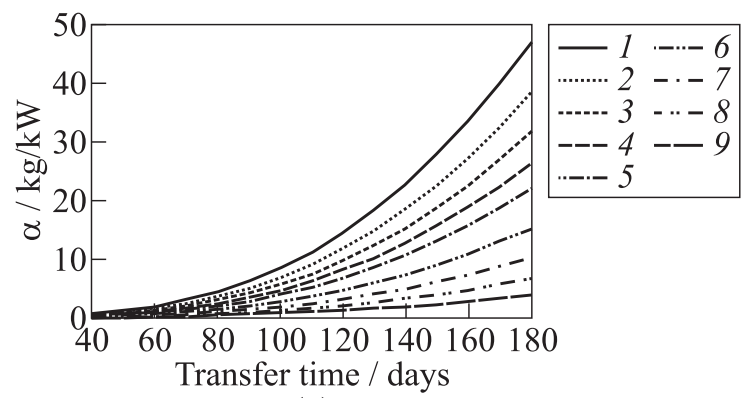

(a)

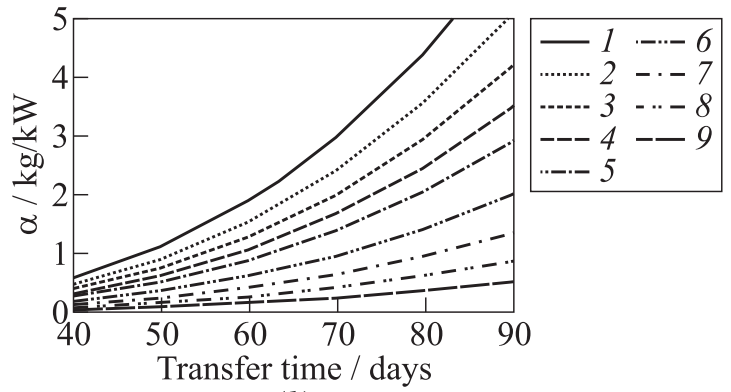

(b)

Figure 5 Specific mass requirements for LEO-to-Mars transfer (global efficiency 0.6 and tank mass ratio 1) - global view for 40- to 180-day transfer times $(a)$ and zoom for short-time missions $(b): 1-M_{\mathrm{pl}} / M_{i}=0 ; 2-0.05 ; 3-0.1 ; 4-0.15 ; 5-0.2$; $6-0.3 ; 7-0.4 ; 8-0.5 ;$ and $9-M_{\mathrm{pl}} / M_{i}=0.6$

calculated. The results are displayed in Figs. 5a (40-180 day transfer) and 5b (zoom for short transfer times under 90 days).

It should be reminded that these results were obtained with the following assumptions (or approximations):

- the vehicle's mass budget was defined in a very simplified way (cf. above);

- for a given mission and given thruster characteristics ( $I_{\mathrm{sp}}$ range), it was assumed that $\lambda$ is a function of the transfer time only and is nearly invariant to the vehicle's sizing variables (power and masses). It was considered that the $\lambda\left(t_{f}\right)$ model of Eq. (8) yields a correct order of magnitude;

- it was assumed that the global efficiency remains constant throughout the thrust arc and does not vary with the level of the specific impulse (this is a condition needed to write Eq. (2) with $\eta$ outside the integral); and

- when considering a single value for the tank structural mass ratio (whatever the dimension and the size of the vehicle), the "scaling" that is typically 
observed for rocket stages and leads to lower values for bigger stages was not taken into account.

Despite these simplifications, the interest of these results is that they give correct orders of magnitude that can be used for preliminary design for a given mission and considering a thruster with a given $I_{\mathrm{sp}}$ range $(3,000-30,000 \mathrm{~s}$ in the case considered in this paper). Also, the role of Eqs. (3) to (6) is to show in a relatively simple way the relations between the key data of the problem (the transfer time, the mass ratio, and the technological hypotheses).

Theses results clearly show that the specific mass requirement (i. e., maximum value possible to make the mission feasible) decreases dramatically for very short transfer times. For instance, a 60-day transfer implies a specific mass lower than $1.5 \mathrm{~kg} / \mathrm{kW}$. For a 40-day transfer time, the specific mass should be lower that $0.5 \mathrm{~kg} / \mathrm{kW}$. The values of the specific masses requirement include not only the power generation system specific mass but also the thrusters specific mass.

It should be emphasized that these results (under the different assumptions stated earlier) are independent of the power level and the masses (taken individually). It is verified that the key parameter with regard to the transfer time is the specific mass of the power and propulsion system, whatever the propulsion technology. The key to a fast interplanetary transfer is a very light power and propulsion system, with a very low specific mass.

In the next section, the level of the specific mass that could be achieved in a relatively short term and a longer term is discussed.

\section{ACHIEVABLE SPECIFIC MASS FOR THE POWER SYSTEM DEPENDING ON TECHNOLOGY}

\subsection{Power Levels and Specific Masses Already Achieved or Accessible in a Relatively Short Term}

Between 1960 and 1992, more than 30 reactors have been used in space. However, their power levels were limited to less than $10 \mathrm{kWe}$. Those reactors were using $\mathrm{Na}-\mathrm{K}$ as a coolant and low efficiency thermoelectric or thermionic technologies for conversion. Specific masses made great progress during this period of time but were still very high: $570 \mathrm{~kg} / \mathrm{kW}$ for Romashka in 1967, $400 \mathrm{~kg} / \mathrm{kW}$ for Buk in 1977 and $180 \mathrm{~kg} / \mathrm{kW}$ for TOPAZ in 1987 [17]. Today, such specific masses are not competitive compared to solar panels when considering an application in the Earth orbit.

At the $100 \mathrm{kWe}$ power level, as shown in [3], the specific mass can be expected to be in the range of 30 to $40 \mathrm{~kg} / \mathrm{kWe}$. Such a specific mass for this power could be achievable using thermoelectric conversion but with a lithium coolant instead 
of a $\mathrm{Na}-\mathrm{K}$ one in order to increase the efficiency by increasing the hot side temperature. Such a system could be developed in a relatively short term.

\subsection{Multimegawatt Power Level}

In terms of power level, given the today's state-of-the-art in space reactors and on submarine reactors, power levels that can be considered for a mid-term mission (in 20 years from now) should not exceed a few tens of megawatt. Higher power levels might be considered as not likely to be available in the same time frame.

For the multimegawatt power level, Brayton or Stirling conversion with a high temperature seems to be the most promising solution [3]. Even if those technologies are less challenging than the Rankine conversion (which could allow further reduction of the specific mass), they still would probably take longer to develop than a $100 \mathrm{kWe}$ liquid metal cooled system using thermo-electricity as proposed in subsection 4.1 .

Table 3 gives some examples of power systems (a reactor, a shield, and a conversion) that have been studied in literature to be used for manned Mars missions (either for a manned spaceship or for a support cargo mission) or other applications. Only the references using solid cores are considered, and this table does not pretend to be exhaustive.

Table 3 Some examples of specific masses (nonexhaustive)

\begin{tabular}{|c|c|c|c|c|c|}
\hline $\begin{array}{l}\text { First } \\
\text { author }\end{array}$ & $\begin{array}{c}\text { Year of } \\
\text { publication }\end{array}$ & $\begin{array}{c}\text { Power level, } \\
\text { MWe }\end{array}$ & $\begin{array}{l}\text { Conversion } \\
\text { cycle }\end{array}$ & $\begin{array}{c}\text { Specific } \\
\text { mass }\end{array}$ & Reference \\
\hline R. J. Cassady & 1988 & 8 & & $8 \mathrm{~kg} / \mathrm{kW}$ & [18] \\
\hline J. S. Clark & 1990 & $\begin{array}{l}4 \text { and } \\
8 \mathrm{MWe}\end{array}$ & Brayton & $10 \mathrm{~kg} / \mathrm{kW}$ & [19] \\
\hline R. D. Rovang & 1992 & 5.5 & Rankine (1300 K) & $6.8 \mathrm{~kg} / \mathrm{kWe}$ & [20] \\
\hline B. Cothran & 1994 & 40 & Brayton & $5.4 \mathrm{~kg} / \mathrm{kW}$ & [21] \\
\hline G. Woodcock & 2002 & 10 & $\begin{array}{c}\text { MHD or Brayton } \\
(1500 \text { to } \\
2000 \mathrm{~K})\end{array}$ & 3.5 to $5 \mathrm{~kg} / \mathrm{kWe}$ & {$[22]$} \\
\hline G. R. Longhurst & 2002 & 15 & $\begin{array}{l}\text { Rankine }(1500 \mathrm{~K}) \\
\text { Brayton }(2100 \mathrm{~K})\end{array}$ & $\begin{array}{l}6.88 \mathrm{~kg} / \mathrm{kWe} \\
2.58 \mathrm{~kg} / \mathrm{kW}\end{array}$ & {$[23]$} \\
\hline J.A. Webb & 2011 & $\begin{array}{c}15 \\
100 \\
1\end{array}$ & Brayton $1300 \mathrm{~K}$ & $\begin{array}{c}15 \mathrm{~kg} / \mathrm{kW} \\
9.6 \mathrm{~kg} / \mathrm{kW} \\
\text { (extrapolated) } \\
32 \mathrm{~kg} / \mathrm{kW}\end{array}$ & {$[24]$} \\
\hline$\overline{R . J . ~ L i t c h f o r d ~}$ & 2011 & $1-5 \mathrm{MWe}$ & MHD (1800K) & 1 to $3 \mathrm{~kg} / \mathrm{kWe}$ & [25] \\
\hline
\end{tabular}


These results show a large dispersion, not only due to the choice of the conversion cycle (the Rankine is lighter than the Brayton because of much smaller radiators). Even though the perimeter included in the specific mass of the system is not homogenous in the publications (some references explicitly take into account power management and distribution (PMAD), structures, and extra margin provisions, but some other references do not give such precision), this does not fully explain the dispersion.

Indeed, the very small specific masses are mainly explained by the hypothesis of the temperature considered as acceptable for both solid cores (very high temperature cores proposed in literature often rely on core technologies developed for nuclear thermal rockets; however, such systems do not have the same specifications in terms of operating duration) and turbines, but it also relies on evaluation of MHD conversion specific mass that is low compared to turbines specific mass. Those points are the subject to large debates [22].

Concerning the authors' position, the ongoing studies [3] tend to suggest that, for the 5-megawatt power level, the specific mass of a Brayton conversion system (cycle and radiators) could be between 6 (for 1600-kelvin turbine inlet temperature) and $10 \mathrm{~kg} / \mathrm{kW}$ (1300-kelvin turbine inlet temperature). Those figures do not include the reactor and shielding mass, neither PMAD. Taking into account all those other items would likely give a total specific mass for the full power generation system that could be nearly doubled compared to the conversion mass.

Thus, considering a Brayton with 1300 to $1600 \mathrm{~K}$ turbine inlet temperature (which are already challenging), the specific mass of a multi-megawatt power system can be expected to be of about $15 \mathrm{~kg} / \mathrm{kW}$ at $5 \mathrm{MW}$. The positive effect of scaling could allow a slight reduction for $20 \mathrm{MW}$ power level (or a lower turbine inlet temperature for the same specific mass objective).

The Rankine cycle could allow further mass reduction (potentially as low as $5 \mathrm{~kg} / \mathrm{kW}$ for the total system at 20 -megawatt power level), but today the feasibility of a space propulsion nuclear reactor operating in zero or very low gravity environment with the Rankine conversion is still under question.

Looking for further reduction of the specific mass $(<3 \mathrm{~kg} / \mathrm{kW})$ would necessarily need an even higher core temperature which makes the feasibility of a solid core quite questionable. It is worth noting that the reduction of the specific mass is mainly coming from the higher operating temperature more than from the magnetohydrodynamic (MHD) converter itself. There is no certitude today that a MHD converter, which needs superconducting magnets and cooling, would be lighter than a turbine. Vapor core reactors have also been considered [26] but their feasibility is even less guaranteed.

Specific mass of the thrusters, not discussed above, has to be added to the power system generation specific mass if it is not taken into account in the tank structural index. 


\section{MISSION ANALYSIS FOR FAST EARTH-MARS TRANSFERS}

\subsection{Definition of the Mission Scenario and Hypotheses}

In this section, first, the feasibility of a very short Earth-to-Mars transfer of 40 days is discussed, then different scenarios that are consistent with the achievable specific mass levels discussed in section 4 are studied.

In all cases, a VASIMR-like propulsion system, whose specific impulse can vary between 3,000 and 30,000 $\mathrm{s}$ is considered. The same hypotheses given in section 3 are put under consideration with regard to the global efficiency (0.6) and the tank and engine structural mass ratio (0.1), which can be regarded as optimistic.

The transfer starts from LEO and ends at Mars with the velocity of $6 \mathrm{~km} / \mathrm{s}$ (for an aerocapture using the atmosphere of Mars).

\subsection{Feasibility of a 40-Day Transfer Scenario}

The order of magnitude of the trajectory characteristic parameter for a 40-day transfer is about $1200 \mathrm{~m}^{2} / \mathrm{s}^{3}$. This order of magnitude has been observed in different mission analysis results from the literature (e.g., [5]) and new trajectory optimization cases performed by CNES (cf. section 2).

With this value of $\lambda$, it is known that the mission can only be achieved with a specific mass lower that $0.5 \mathrm{~kg} / \mathrm{kW}$, which is the theoretical maximum value considering the "extreme" case of a null payload mass. As explained in section 3, such a low value appears extremely optimistic, and its feasibility is questionable even with the most advanced technologies currently considered, unless in a very long term.

Before studying more realistic scenarios in subsection 5.3, an example is provided of the optimal design for a 40-day mission derived from the 39 days LEOto-Mass transfer first studied by Ad Astra for its VASIMR concept (cf. [8]).

First, let remind the original Ad Astra vehicle design for the 39 day LEO to Mars transfer, as defined in [8]:

- power source: $P_{e}=200 \mathrm{MW}$; and

- mass budget — see Table 4.

The specific mass (as defined in this paper) for this case is $\alpha \sim 0.4 \mathrm{~kg} / \mathrm{kW}$ and the payload mass ratio is 0.0325

As shown by equations of subection 3.2 , the maximum payload mass ratio that can be obtained with the same values of $\lambda$ and $\alpha$ is 0.0616 . 
Table 4 Mass budget for Ad Astra's fast Earth-to-Mars transfer from LEO (as defined in [8])

\begin{tabular}{lcc}
\hline \multicolumn{1}{c}{ Type } & Mass, t & Remarks \\
\hline Payload at Mars $\left(M_{\mathrm{pl}}\right)$ & 20 & Fixed \\
\hline $\begin{array}{l}\text { Mass of power } \\
\text { and propulsion system }\left(M_{T}\right)\end{array}$ & 79.5 & $\begin{array}{l}\text { Computed by: } \\
M_{P}=M_{i}-M_{P}-M_{S}-M_{\mathrm{pl}}\end{array}$ \\
\hline Propellant mass $\left(M_{P}\right)$ & 455 & $\begin{array}{c}\text { Obtained through trajectory } \\
\text { optimization (Copernicus) }\end{array}$ \\
\hline Tank structure mass $\left(M_{S}\right)$ & 45.5 & Defined through $k=M_{S} / M_{P}=0.1$ \\
\hline \hline Total initial mass in LEO $\left(M_{i}\right)$ & 600 & \\
\hline
\end{tabular}

Table 5 Mass budget of the optimum design for the 39 day / 20 t payload mission

\begin{tabular}{lcc}
\hline \multicolumn{1}{c}{ Type } & Mass, t & Remarks \\
\hline Payload at mars $\left(M_{\mathrm{pl}}\right)$ & 20.6 & Fixed \\
\hline $\begin{array}{l}\text { Mass of power } \\
\text { and propulsion system }\left(M_{T}\right)\end{array}$ & 66.2 & Computed by: $M_{T}=\alpha P_{e}$ \\
\hline Propellant mass $\left(M_{P}\right)$ & 148.2 & $\begin{array}{c}\text { Obtained through } \\
\text { trajectory optimization }\end{array}$ \\
\hline Tank structure mass $\left(M_{S}\right)$ & 14.8 & Defined through $k=M_{S} / M_{P}=0.1$ \\
\hline \hline Total initial mass in LEO $\left(M_{i}\right)$ & 250 & \\
\hline
\end{tabular}

Using a simple iterative design process based on the optimal design equations of subsection 3.2 and trajectory optimizations (cf. section 2), the best vehicle design that can perform the same mission in terms of the payload mass $(20 \mathrm{t}$ ) and the transfer time (39 days) with the minimum total initial mass was calculated. The characteristics are the following:

- power source: $P_{e}=165.6 \mathrm{MW}$; and

- mass budget - see Table 5 .

The payload mass ratio for this case is 0.0830 and the total initial mass in LEO is $250 \mathrm{t}$ instead of $600 \mathrm{t}$, which is a significant improvement. This optimum design is characterized by a power-to-total mass ratio $P_{e} / M_{i}=662.4 \mathrm{~W} / \mathrm{kg}$ (instead of $333.3 \mathrm{~W} / \mathrm{kg}$ in the original design).

The updated value of the trajectory characteristic parameter is $\lambda$ $=1187.5 \mathrm{~m}^{2} / \mathrm{s}^{3}$ (this value is an output of the trajectory optimization).

This study illustrates the order of magnitude of the maximum payload mass ratio that can be obtained for a 40-day Earth-to-Mars transfer. Nevertheless, it 
should be reminded that the feasibility of such a fast transfer depends - in any case - on a very demanding requirement in terms of the specific mass.

\subsection{Proposition of Scenarios Achievable at Shorter Term}

Following the considerations exposed in section 4 about the possible improvements of nuclear power technologies, different mission scenarios have been studied and their feasibility has been tested for two different specific mass hypotheses:

(1) a "reasonably optimistic" hypothesis with $\alpha=10 \mathrm{~kg} / \mathrm{kW}$, which corresponds to what can be expected in the future with the Brayton cycle technology; and

(2) a more optimistic "long term" hypothesis with $\alpha=5 \mathrm{~kg} / \mathrm{kW}$, that could be achieved using the Rankine cycle technology at a longer term (cf. [3]).

The tank structural mass ratio is taken equal to $10 \%$ of the propellant mass. Since, the above mentioned specific masses do not include a thrusters mass, in this particular case we will consider that the structural index includes it.

Table 6 corresponds to the payload that can be obtained with these hypotheses of specific masses for different trajectory optimization cases with a power level of $20 \mathrm{MW}$. The data for these cases are extracted from the results of section 2 (cf. Figs. 3 and 4).

It should be noted that, according to the technological hypotheses (represented by $k$ and $\alpha$ ), the payload mass obtained may be negative.

Table 6 Trajectory optimization results and payload masses obtained for $\alpha=5$ and $10 \mathrm{~kg} / \mathrm{kW}$ and $20 \mathrm{MW}$

\begin{tabular}{ccccccc}
\hline & \multicolumn{3}{c}{$\begin{array}{c}\text { Total final } \\
\text { mass, } \mathrm{t},\end{array}$} \\
\cline { 4 - 7 } $\begin{array}{c}\text { Trajectory } \\
\text { case } \\
\text { number }\end{array}$ & $\begin{array}{c}\text { Transfer } \\
\text { time, } \\
\text { days }\end{array}$ & $\begin{array}{c}\text { IMLEO, } \\
\text { with arrival } \\
\text { velocity } \\
\text { at Mars } \\
\text { of } 6.8 \mathrm{~km} / \mathrm{s}\end{array}$ & $\begin{array}{c}\lambda, \\
\mathrm{m}^{2} / \mathrm{s}^{3}\end{array}$ & $\alpha=5 \mathrm{~kg} / \mathrm{kW}$ & $\alpha=10 \mathrm{~kg} / \mathrm{kW}$ \\
\hline 1 & 90 & 200 & 112 & 94.3 & $\mathbf{3 . 2}$ & $(-96.8)$ \\
2 & 90 & 300 & (no solution) & & & \\
3 & 90 & 400 & (no solution) & & & \\
4 & 120 & 200 & 136 & 56.5 & $\mathbf{3 0}$ & $(-70.4)$ \\
5 & 120 & 300 & 186 & 49.0 & $\mathbf{7 5}$ & $(-25.4)$ \\
6 & 120 & 400 & 200 & 60 & $\mathbf{8 0}$ & $(-20.0)$ \\
7 & 180 & 500 & 329 & 94.3 & $\mathbf{2 1 2}$ & $\mathbf{1 1 2}$ \\
\hline
\end{tabular}


Results are discussed separately for the different transfer times considered in the following paragraphs. In addition, all the feasible scenarios of Table 6 (i. e., scenarios with a positive payload mass) are displayed in the $\left[\lambda, M_{P L} / M_{i}\right]$ domain on Fig. 6 . The figure also displays the curves of the maximum payload mass ratio as a function of $\lambda$, as calculated with Eq. (3), for different values of the specific mass, including 5 and $10 \mathrm{~kg} / \mathrm{kW}$ (curves 4 and 5 ). This graphic makes possible to compare the different design cases to the corresponding optimum design (which yields the maximum payload mass ratio with the same $\lambda$ value). It should be reminded, however, that $\lambda$ has not exactly the same value for different cases with the same transfer time, but the order of magnitude remains the same at isotransfer time.

90 day-mission. Trajectory optimization failed to find 90 day-transfer solutions for the test cases with an IMLEO of 300 and $400 \mathrm{t}$.

The only feasible case is the one with a $200 \mathrm{t}$ IMLEO, which yields a total final mass of $112 \mathrm{t}$. This mass is insufficient to have a positive payload mass with the Brayton hypothesis $(\alpha=10 \mathrm{~kg} / \mathrm{kW})$. On the other hand, the optimistic Rankine hypothesis $(\alpha=5 \mathrm{~kg} / \mathrm{kW})$ allows a positive payload mass, although it is very small (3.2 t).

Using Eq. (7) and the same value of $\lambda$, it can be shown that the theoretical maximum value of the specific mass that makes the mission feasible is around 6-7 kg/kW, which is consistent with these results.

Considering the Rankine hypothesis $(\alpha=5 \mathrm{~kg} / \mathrm{kW})$, the payload mass is $3.2 \mathrm{t}$, which corresponds to a 0.016 mass ratio.

The theoretical maximum mass ratio that can obtained with the same trajectory characteristic $\alpha\left(\sim 100 \mathrm{~m}^{2} / \mathrm{s}^{3}\right)$ using Eq. (2) is 0.078 (cf. curve 4 in Fig. 6 ), which represents the optimum design for $\alpha=5 \mathrm{~kg} / \mathrm{kW}$ ). This result suggests that the payload mass could be multiplied by a factor 4 or 5 with an appropriate vehicle sizing. However, this would yield only a payload mass of about 10-15 t at best with the same IMLEO (200 t), which appears to be still insufficient for a manned Mars mission. Moreover, the feasibility of such mission depends on a very optimistic hypothesis regarding the specific mass.

120-day mission. Feasible optimal trajectories could be obtained for all of three IMLEO cases $(200,300$ and 400 t), with final total masses between 136 and 200 t. Again, these final masses make the mission actually feasible for the Rankine hypothesis $(\alpha=5 \mathrm{~kg} / \mathrm{kW})$ only, while the payload mass is negative with the Brayton hypothesis $(10 \mathrm{~kg} / \mathrm{kW})$.

Considering $\alpha=5 \mathrm{~kg} / \mathrm{kW}$, the payload mass obtained in the three IMLEO cases are 30,75 , and $80 \mathrm{t}$, which corresponds to mass ratios of $0.148,0.249$, and 0.20 , respectively. Calculations of theoretical optimal mass ratios show that the vehicle designs for the 300- and 400-ton IMLEO cases are nearly optimal (this 


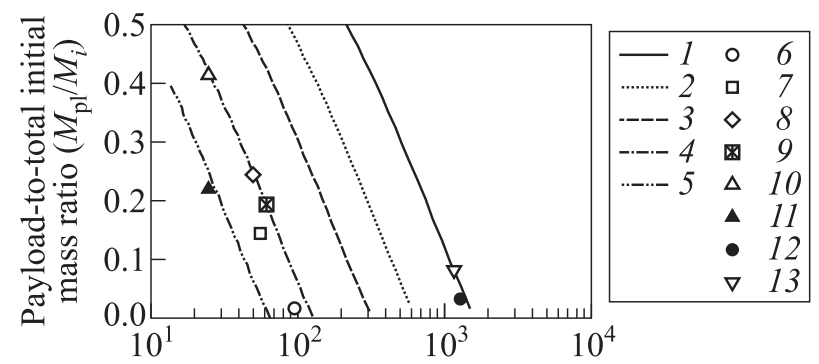

Trajectory characteristic $\lambda / \mathrm{m}^{2} / \mathrm{s}^{3}$

Figure 6 Test-cases summary and comparison with theoretical optimum vehicle sizing for different specific masses: $1-$ optimum, $\alpha=0.4 \mathrm{~kg} / \mathrm{kW} ; 2-1 ; 3-2 ; 4-5$; and $5-$ optimum, $\alpha=10 \mathrm{~kg} / \mathrm{kW} ; \alpha=5 \mathrm{~kg} / \mathrm{kW}: 6-90$ days, $200 \mathrm{t} ; 7-120$ days, $200 \mathrm{t}, 8-120$ days, $300 \mathrm{t} ; 9-120$ days, $400 \mathrm{t}$; and $10-180$ days, $500 \mathrm{t} ; 11-$ $\alpha=10 \mathrm{~kg} / \mathrm{kW}, 180$ days, $500 \mathrm{t} ; 12$ - original 39-day mission; and 13 - optimized 39-day mission

can be checked in Fig. 6) through the proximity of the design points and the optimal design curves for $\alpha=5 \mathrm{~kg} / \mathrm{kW}$ ).

The 300-ton case appears to be the best of these two cases since its payload mass $(75 \mathrm{t})$ shows a 5 -ton only penalty compared to the one of the 400 -ton case, even though the IMLEO is $100 \mathrm{t}$ smaller.

Unlike the 90-day mission for which the payload was too small, the payload for the 120-day mission is definitely relevant for a manned mars mission $(\sim 75$ $80 \mathrm{t}$ ). Again, it should be reminded that the feasibility of this mission depends on the "optimistic" Rankine hypothesis $(\alpha=5 \mathrm{~kg} / \mathrm{kW})$.

Actually, the theoretical maximum value for the specific mass considering a null payload mass and the same values of $\lambda$ obtained $\left(\sim 50-60 \mathrm{~m}^{2} / \mathrm{s}^{3}\right)$ is about $12 \mathrm{~kg} / \mathrm{kW}$, which is a little higher that the value we fixed for the Brayton hypothesis. This suggests that a 120 day-mission may actually be feasible in this case, but with a much smaller mass ratio of around 0.02 to 0.07 . Such values appear to be inappropriate for a manned Mars mission as they lead to a very high IMLEO.

180-day mission. Only one IMLEO case (500 t) has been tested. The trajectory optimization yields a 328 ton total final mass, which makes the mission feasible for both the 5 and the $10 \mathrm{~kg} / \mathrm{kW}$ hypotheses. The payload masses obtained are 212 and 112 t, which correspond to mass ratios of 0.42 and 0.22 , respectively. These payload masses are definitely relevant for a manned Mars mission, although it should be noted that the IMLEO is high $(500 \mathrm{t})$.

In both cases, the current vehicle designs have shown to be nearly optimal in terms of the payload mass ratio (cf. Fig. 6), which means that the 
IMLEO cannot probably be reduced any further considering the same payload masses.

\section{CONCLUDING REMARKS}

The simplified performance model for Power-Limited systems presented in section 3 has made it possible to obtain a global view of the relations between the transfer time, the payload mass ratio and the technical characteristics of the power and propulsion system. Although many approximations are made in this model, it has been proved to be an efficient tool to get the optimum design in terms of the payload mass ratio for a given transfer time. Using this model, it was verified that the key parameter of fast interplanetary transfers is the specific mass of the power and propulsion system, which should be lower than about $0.5 \mathrm{~kg} / \mathrm{kW}$ for 40 -day LEO-to-Mars transfer. This requirement is clearly incompatible with the level of specific mass that could be achieved at short/medium mission term, which is about $10 \mathrm{~kg} / \mathrm{kW}$ considering the Braytoncycle technology. The mission analysis study performed in section 3 has shown that this "reasonably optimistic" hypothesis for the specific mass does not enable a significant reduction of the transfer time.

At a farther term, a specific mass of about $5 \mathrm{~kg} / \mathrm{kW}$ may be achieved using a more advanced technology (the Rankine-cycle technology). With this value for the specific mass, a 120-day transfer can be achieved with interesting performance in terms of the mass (IMLEO of $300 \mathrm{t}$ and payload mass about $80 \mathrm{t}$ ). This represents a significant reduction of the transfer time compared to current scenarios with conventional propulsion technologies (typically 180 days) but this reduction is moderate compared to the objective of a very fast transfer (e.g., 40 days).

With the same hypothesis $(5 \mathrm{~kg} / \mathrm{kW})$, a transfer time shorter that 120 days can actually be achieved but at the cost of a dramatic reduction of the payload mass, which makes the mission irrelevant (e.g., 3-ton payload mass for a 90 -day transfer starting from a 200-ton IMLEO).

\section{REFERENCES}

1. Chang-Diaz, F. R., M. M. Hsu, E. Braden, I. Johson, and T. Fang-Yang. 1995. Rapid Mars transits with exhaust-modulated plasma propulsion. NASA Technical Paper 3539.

2. Berend, N. 2012. Optimum design of power-limited propulsion systems with application to fast-Earth-to-Mars transfer. Acta Astronautica 79:107-17. 
3. Cliquet, E., J. M. Ruault, J. P. Roux, L. Lamoine, N. Paris, and B. Cazale. 2011. Study of space reactors for exploration missions. 4th European Conference for Aerospace Sciences. Saint-Petersburg, Russia.

4. Ragsac, R.V. 1967. Study of electric propulsion for manned Mars missions. J. Spacecraft 4(4).

5. Sankaran, K., L. Cassady, A.D. Kodys, and E. Y. Choueiri. 2003. A survey of propulsion options for cargo and piloted missions to Mars. Conference (International) on New Trends in Astrodynamics.

6. Landau, D. F., and J. M. Longuski. 2006. Trajectories for human missions to Mars. Part 2: Low-thrust transfers. J. Spacecrafts Rockets 43(5).

7. Schmidt, T. D., W. Seboldt, and M. Auweter-Kurtz. 2006. Flexible piloted Mars missions using continuous electric propulsion. J. Spacecrafts Rockets 43(6).

8. Ilin, V, L. Cassady, T. Glover, M. Carter, and F. Chang-Diaz. 2010. A survey of missions using VASIMR for Flexible Space Exploration. NASA Technical Report JSC-65828.

9. Kodys, A.D., and E. Y. Choueiri. 2005. A critical review of the state-of-the-art in the performance of applied-field magnetoplasmadynamic thrusters. AIAA Joint Propulsion Conference.

10. Dankanich, J. W., B. Vondra, and A. V. Illin. 2010. Fast transit to Mars using electric propulsion. AIAA Paper No. 2010-6771.

11. Irving, J. 1959. Low-thrust flight: Variable exhaust velocity in gravitational fields. In: Space Technology. Ed. H. S. Seifert, Wiley. Ch. 10.

12. Melbourne, W. G. 1961. Interplanetary trajectories and payload capabilities of advanced propulsion vehicle. JPL Technical Report No. 32-68.

13. Melbourne, W. G., and C. G. Sauer. 1963. Payload optimization for power-limited vehicles. Electric Propulsion Conference. Berkeley, CA.

14. Ragsac, R. V. 1968. Handbook for the study of electrical propulsion systems. Report G-110058-28. United Aircraft Laboratory.

15. Glover, T. G. 2004. VASIMR: Express flight to Mars. MarsWeek Conference, MIT.

16. Illin, A. V., L.D. Cassady, T.W. Glover, and F. R. Chang Diaz. 2011, March. VASIMR human mission to Mars. Space Propulsion and Energy Sciences International Forum (SPESIF 2011). College Park, MD.

17. Graham, J., V. Ionkin, and N. N. Ponomarev-Stepnoi. 2005. The role of nuclear power and nuclear propulsion in the peaceful exploration of space. IAEA.

18. Cassady, R. J. 1988. An MPD thrusters driven cargo ferry for support of the manned Mars mission. AIAA Paper No. 88-2896.

19. Clark, J. S., J.A. George, and L.P. Gefert. 1994. Nuclear electric propulsion, a "Better, Safer, Cheaper" transportation system for human exploration of Mars. 11th Symposium on Space Nuclear Power and Propulsion Systems. Albuquerque New Mexico. NASA TM 106406.

20. Rovang, R.D., G. A. Johnson, and J. C. Mills. 2002. Potassium-Rankine nuclear electric propulsion for Mars cargo missions. STAIF 2002.

21. Cothran, B., B. Tillotson, and B. Donahue. 1990. Advanced options for human exploration of Mars. AIAA Paper No. 90-3822. 
22. Woodcock, G. 2002. Evaluation of high performance space nuclear electric generators for electric propulsion application. STAIF 2002.

23. Longhurst, G. R., B. G. Schnitzler, and B. T. Parks. 2002. Multi-Megawatt Power system trade study. STAIF 2002.

24. Webb, J. A., and J. Brian. 2011. Gross, conceptual multimegawatt system based on a tungsten CERMET reactor. NETS 2011.

25. Litchford, R. J., and N. Harada. 2011. Multi MW closed cycle MHD nuclear space power via nonequilibrium $\mathrm{He} / \mathrm{Xe}$ frozen inert plasma. NETS 2011.

26. Smith, B., T. Knight, and S. Anghaie. 2002. Multimegawatt NEP with vapour core reactor MHD. STAIF 2002. 